\title{
Stability Analysis of Time-Delay Systems by Nonlinear Approximation
}

\author{
A. Yu. Aleksandrov, E. B. Aleksandrova, A. P. Zhabko \\ Paper dedicated to memory of Vera Nikolić-Stanojević, \\ Pofessor Emeritus at the State University of Novi Pazar
}

\begin{abstract}
We consider a nonlinear complex (large-scale) system with time delay. It is assumed that the corresponding isolated subsystems are homogeneous, and the zero solutions of the subsystems are asymptotically stable when delay is equal to zero. By the Lyapunov direct method, and the Razumikhin approach, delay-independent stability conditions for the complex system are obtained. These conditions are formulated in terms of solvability of auxiliary systems of algebraic inequalities. An example is given to demonstrate effectiveness of the presented results.

Keywords: Complex systems, delay, asymptotic stability, Lyapunov functions
\end{abstract}

\section{Introduction}

The problem of stability of nonlinear time-delay systems has been studied intensively during the past decades, see, for example, $[3,6,7]$. Here there are two principal approaches, the first one is based on the Lyapunov-Krasovskii functionals, the other one is based on the Razumikhin theorem. These approaches were successfully applied by various authors to the study of wide classes of systems, see $[2-4,6,7]$ and the references cited therein.

Stability conditions may depend on delay, and in this case, we cannot guarantee that the system remains stable when delay exceeds a certain value. In some applications, the smallness of delay cannot be assured. Moreover, the delay value may be unknown. Therefore, it is important to have stability conditions under which the system remains stable for any positive value of delay. Such conditions are known as delay-independent ones [3].

In this paper, a nonlinear complex (large-scale) system with time delay is considered. It is assumed that the corresponding isolated subsystems are homogeneous, and the zero solutions of the subsystems are asymptotically stable when delay is equal to zero. By the

Manuscript received June 17, 2014. ; accepted October 4, 2014.

A. Yu. Aleksandrov, E. B. Aleksandrova, and A. P. Zhabko are with the Faculty of Applied Mathematics and Control Processes, Saint Petersburg State University, Saint Petersburg, Russia. 
Lyapunov direct method, and the Razumikhin approach, delay-independent stability conditions for the complex system are obtained. These conditions are formulated in terms of solvability of auxiliary systems of algebraic inequalities. An example is given to demonstrate effectiveness of the presented results.

\section{Statement of the Problem}

In the sequel, $\mathbb{R}$ denotes the field of real numbers, and $\mathbb{R}^{n}$ the $n$-dimensional Euclidean space. For a given real number $\tau \in(0,+\infty)$, let $C\left([-\tau, 0], \mathbb{R}^{n}\right)$ be the space of continuous functions $\varphi(\theta):[-\tau, 0] \rightarrow \mathbb{R}^{n}$. The Euclidean norm will be used for vectors. For elements of the space $C\left([-\tau, 0], \mathbb{R}^{n}\right)$ we will use the uniform norm $\|\varphi\|_{\tau}=\max _{\theta \in[-\tau, 0]}\|\varphi(\theta)\|$.

Consider the system

$$
\dot{\mathbf{z}}_{s}(t)=\mathbf{f}_{s}\left(\mathbf{z}_{s}(t), \mathbf{z}_{s}(t-\tau)\right)+\sum_{j=1}^{m} \mathbf{Q}_{s j}\left(t, \mathbf{x}_{t}\right), \quad s=1, \ldots, m .
$$

Here $\mathbf{x}(t)=\left(\mathbf{z}_{1}^{T}(t), \ldots, \mathbf{z}_{m}^{T}(t)\right)^{T} \in \mathbb{R}^{n}$ is the state vector, $\mathbf{z}_{s}(t) \in \mathbb{R}^{n_{s}}, n=n_{1}+\ldots+n_{m} ; \tau>0$ is a constant delay; the components of the vectors $\mathbf{f}_{s}\left(\mathbf{z}_{s}, \mathbf{u}_{s}\right)$ are homogeneous functions of the orders $\mu_{s}>1$, defined for $\mathbf{z}_{s}, \mathbf{u}_{s} \in \mathbb{R}^{n_{s}}$, and are continuous with respect to their variables, and continuously differentiable with respect to $\mathbf{u}_{s}$; the functionals $\mathbf{Q}_{s j}(t, \varphi)$ are given and continuous in the domain

$$
\{t \in \mathbb{R}: t \geq 0\} \times \Omega_{H},
$$

where $\Omega_{H}$ is the set of functions $\varphi(\theta) \in C\left([-\tau, 0], \mathbb{R}^{n}\right)$ satisfying the inequality $\|\varphi\|_{\tau}<H$, $0<H \leq+\infty$. Moreover, we assume that the estimates

$$
\left\|\mathbf{Q}_{s j}(t, \varphi)\right\| \leq \beta_{s j}\left(\left\|\psi_{j}\right\|_{\tau}\right)^{\alpha_{s j}}, \quad s, j=1, \ldots, m,
$$

are valid in the domain (2). Here $\varphi(\theta)=\left(\psi_{1}^{T}(\theta), \ldots, \psi_{m}^{T}(\theta)\right)^{T}, \psi_{j}(\theta) \in C\left([-\tau, 0], \mathbb{R}^{n_{j}}\right)$, and $\beta_{s j} \geq 0, \alpha_{s j}>1 ; s, j=1, \ldots, m$. Thus, system (1) admits the zero solution.

Let $\mathbf{x}\left(t, t_{0}, \varphi\right)$ stand for a solution of system (1) with the initial conditions $t_{0} \geq 0, \varphi(\theta) \in$ $\Omega_{H}$, and $\mathbf{x}_{t}\left(t_{0}, \varphi\right)$ denote the restriction of the solution to the segment $[t-\tau, t]$, i.e., $\mathbf{x}_{t}\left(t_{0}, \varphi\right)$ : $\theta \rightarrow \mathbf{x}\left(t+\theta, t_{0}, \varphi\right), \theta \in[-\tau, 0]$. In some cases, when the initial conditions are not important, or are well defined from the context, we write $\mathbf{x}(t)$ and $\mathbf{x}_{t}$, instead of $\mathbf{x}\left(t, t_{0}, \varphi\right)$ and $\mathbf{x}_{t}\left(t_{0}, \varphi\right)$, respectively.

System (1) describes the dynamics of a complex system composed of $m$ interconnected subsystems [9]. The functions $\mathbf{f}_{s}\left(\mathbf{z}_{s}, \mathbf{u}_{s}\right)$ define the interior connections of subsystems, whereas the functionals $\mathbf{Q}_{s j}(t, \varphi)$ characterize the interaction between the subsystems.

Assumption 1. Let the zero solutions of the isolated delay free subsystems

$$
\dot{\mathbf{z}}_{S}(t)=\mathbf{f}_{s}\left(\mathbf{z}_{S}(t), \mathbf{z}_{s}(t)\right), \quad s=1, \ldots, m,
$$

be asymptotically stable. 
We will look for conditions under which the zero solution of (1) is also asymptotically stable for an arbitrary value of $\tau>0$.

In [5], an approach for the stability analysis of essentially nonlinear delay free complex systems was suggested. In [1], the results of [5] were strengthened and extended to wider classes of systems. In the present paper, we will show that the approaches developed in [1, 5] permit to obtain delay-independent stability conditions for complex system (1).

\section{Asymptotic Stability Conditions}

Consider the first auxiliary system of inequalities

$$
\alpha_{s j} h_{j} \geq \mu_{s} h_{s}, \quad s, j=1, \ldots, m .
$$

Assumption 2. System (4) admits positive solutions.

Assumption 3. For subsystems (3), Lyapunov functions $V_{1}\left(\mathbf{z}_{1}\right), \ldots, V_{m}\left(\mathbf{z}_{m}\right)$ are constructed, such that $V_{s}\left(\mathbf{z}_{s}\right)$ is continuously differentiable for $\mathbf{z}_{s} \in \mathbb{R}^{n_{s}}$ positive definite and homogeneous of the degree $\gamma_{s}>1$ function, and the derivative of $V_{s}\left(\mathbf{z}_{s}\right)$ with respect to $s$-th subsystem from the family (3) is negative definite, $s=1, \ldots, m$.

Assumption 4. The numbers $h_{s}=1 /\left(\gamma_{s}+\mu_{s}-1\right), s=1, \ldots, m$, satisfy the inequalities (4).

Remark 1. In $[8,10]$ it is proved that the fulfilment of Assumption 1 implies the existence of the required Lyapunov functions.

Remark 2. In view of the homogeneous functions properties, see [10], the estimates

$$
\begin{gathered}
a_{1 s}\left\|\mathbf{z}_{s}\right\|^{\gamma_{s}} \leq V_{s}\left(\mathbf{z}_{s}\right) \leq a_{2 s}\left\|\mathbf{z}_{s}\right\|^{\gamma_{s}} \\
\left\|\frac{\partial V_{s}}{\partial \mathbf{z}_{s}}\right\| \leq a_{3 s}\left\|\mathbf{z}_{s}\right\|^{\gamma_{s}-1}, \quad\left(\frac{\partial V_{s}}{\partial \mathbf{z}_{s}}\right)^{T} \mathbf{f}_{s}\left(\mathbf{z}_{s}, \mathbf{z}_{s}\right) \leq-a_{4 s}\left\|\mathbf{z}_{s}\right\|^{\gamma_{s}+\mu_{s}-1}
\end{gathered}
$$

hold for all $\mathbf{z}_{s} \in \mathbb{R}^{n_{s}}$, where $a_{1 s}, a_{2 s}, a_{3 s}, a_{4 s}$ are positive constants, $s=1, \ldots, m$.

Next, consider the second auxiliary system of inequalities

$$
-a_{4 s} \eta_{s}^{\mu_{s}}+a_{3 s} \sum_{j=1}^{m} \beta_{s j} \eta_{j}^{\alpha_{s j}}<0, \quad s=1, \ldots, m
$$

Remark 3. Coefficients $a_{3 s}$ and $a_{4 s}, s=1, \ldots, m$, in (5) depend on the constructed Lyapunov functions $V_{1}\left(\mathbf{z}_{1}\right), \ldots, V_{m}\left(\mathbf{z}_{m}\right)$.

Assumption 5. System (5) admits positive solutions.

Theorem 1 Let Assumptions 1-5 be fulfilled. Then the zero solution of system (1) is asymptotically stable for an arbitrary value of $\tau>0$. 
Proof. Choose a Lyapunov function for system (1) in the form

$$
V(\mathbf{x})=\sum_{s=1}^{m} \lambda_{s} V_{s}\left(\mathbf{z}_{s}\right)
$$

where $\lambda_{1}, \ldots, \lambda_{m}$ are positive coefficients. Function (6) is positive definite.

Differentiating $V(\mathbf{x})$ with respect to system (1), we obtain

$$
\begin{aligned}
\left.\dot{V}\right|_{(1)}=\sum_{s=1}^{m} & \lambda_{s}\left(\frac{\partial V_{s}\left(\mathbf{z}_{s}(t)\right)}{\partial \mathbf{z}_{s}}\right)^{T} \mathbf{f}_{s}\left(\mathbf{z}_{s}(t), \mathbf{z}_{s}(t)\right)+\sum_{s=1}^{m} \lambda_{s}\left(\frac{\partial V_{s}\left(\mathbf{z}_{s}(t)\right)}{\partial \mathbf{z}_{s}}\right)^{T} \sum_{j=1}^{m} \mathbf{Q}_{s j}\left(t, \mathbf{x}_{t}\right) \\
& +\sum_{s=1}^{m} \lambda_{s}\left(\frac{\partial V_{s}\left(\mathbf{z}_{s}(t)\right)}{\partial \mathbf{z}_{s}}\right)^{T}\left(\mathbf{f}_{s}\left(\mathbf{z}_{s}(t), \mathbf{z}_{s}(t-\tau)\right)-\mathbf{f}_{s}\left(\mathbf{z}_{s}(t), \mathbf{z}_{s}(t)\right)\right) \\
\leq & \sum_{s=1}^{m} \lambda_{s}\left\|\mathbf{z}_{s}(t)\right\|^{\gamma_{s}-1}\left(-a_{4 s}\left\|\mathbf{z}_{s}(t)\right\|^{\mu_{s}}+a_{3 s} \sum_{j=1}^{m} \beta_{s j}\left\|\mathbf{z}_{j}(t)\right\|^{\alpha_{s j}}\right) \\
& +\sum_{s=1}^{m} \lambda_{s} a_{3 s}\left\|\mathbf{z}_{s}(t)\right\|^{\gamma_{s}-1} \sum_{j=1}^{m} \beta_{s j} \mid\left(\left\|\mathbf{z}_{j t}\right\| \tau\right)^{\alpha_{s j}}-\left\|\mathbf{z}_{j}(t)\right\|^{\alpha_{s j} \mid} \\
& +\sum_{s=1}^{m} \lambda_{s} a_{3 s}\left\|\mathbf{z}_{s}(t)\right\|^{\gamma_{s}-1}\left\|\mathbf{f}_{s}\left(\mathbf{z}_{s}(t), \mathbf{z}_{s}(t-\tau)\right)-\mathbf{f}_{s}\left(\mathbf{z}_{s}(t), \mathbf{z}_{s}(t)\right)\right\|
\end{aligned}
$$

It is known, see [1], that the fulfilment of Assumptions 2, 4 and 5 implies the existence of positive numbers $\tilde{a}, \delta_{0}, \lambda_{1}, \ldots, \lambda_{m}$ such that

$$
\sum_{s=1}^{m} \lambda_{s}\left\|\mathbf{z}_{s}\right\|^{\gamma_{s}-1}\left(-a_{4 s}\left\|\mathbf{z}_{s}\right\|^{\mu_{s}}+a_{3 s} \sum_{j=1}^{m} \beta_{s j}\left\|\mathbf{z}_{j}\right\|^{\alpha_{s j}}\right) \leq-\tilde{a} \sum_{s=1}^{m}\left\|\mathbf{z}_{s}\right\|^{\gamma_{s}+\mu_{s}-1}
$$

for $\|\mathbf{x}\|<\delta_{0}$

Choose a positive integer $l$ and a number $\delta \in\left(0, \delta_{0}\right)$. Assume that, for a solution $\mathbf{x}(t)$ of (1), the inequality $\|\mathbf{x}(\xi)\|<\delta$, and the Razumikhin condition $V(\mathbf{x}(\xi)) \leq 2 V(\mathbf{x}(t))$ are fulfilled for $\xi \in[t-l \tau, t]$. Then

$$
\left\|\mathbf{z}_{s}(\xi)\right\| \leq c_{s} \sum_{i=1}^{m}\left\|\mathbf{z}_{i}(t)\right\|^{\frac{\gamma_{i}}{\gamma_{s}}}, \quad s=1, \ldots, m,
$$

for $\xi \in[t-l \tau, t]$, where $c_{1}, \ldots, c_{m}$ are positive constants.

With the aid of the Mean Value Theorem, it is easy to show that

$$
\begin{gathered}
\left\|\mathbf{f}_{s}\left(\mathbf{z}_{s}(t), \mathbf{z}_{s}(t-\tau)\right)-\mathbf{f}_{s}\left(\mathbf{z}_{s}(t), \mathbf{z}_{s}(t)\right)\right\|+\sum_{j=1}^{m} \beta_{s j}\left|\left(\left\|\mathbf{z}_{j t}\right\|_{\tau}\right)^{\alpha_{s j}}-\left\|\mathbf{z}_{j}(t)\right\|^{\alpha_{s j}}\right| \\
\leq \rho_{s}\left(\left\|\mathbf{z}_{s}(t)\right\|^{\mu_{s}-1}\left(\chi_{s}(t)+\omega_{s}(t)\right)+\left(\chi_{s}(t)+\omega_{s}(t)\right)^{\mu_{s}}\right.
\end{gathered}
$$




$$
\left.+\sum_{j=1}^{m}\left(\left\|\mathbf{z}_{j}(t)\right\|^{\alpha_{s j}-1}\left(\chi_{j}(t)+\omega_{j}(t)\right)+\left(\chi_{j}(t)+\omega_{j}(t)\right)^{\alpha_{s j}}\right)\right), \quad s=1, \ldots, m
$$

Here

$$
\begin{gathered}
\chi_{s}(t)=\left\|\mathbf{z}_{s}(t)\right\|^{\mu_{s}}+\sum_{k=1}^{m}\left\|\mathbf{z}_{k}(t)\right\|^{\alpha_{s k}}, \\
\omega_{s}(t)=\left(\left\|\mathbf{z}_{s t}\right\|_{2 \tau}\right)^{\mu_{s}}-\left\|\mathbf{z}_{s}(t)\right\|^{\mu_{s}}+\sum_{k=1}^{m}\left(\left(\left\|\mathbf{z}_{k t}\right\|_{2 \tau}\right)^{\alpha_{s k}}-\left\|\mathbf{z}_{k}(t)\right\|^{\alpha_{s k}}\right),
\end{gathered}
$$

and $\rho_{s}=$ const $>0, s=1, \ldots, m$. Further, for the functions $\omega_{1}(t), \ldots, \omega_{m}(t)$ the similar estimates can be found.

Successively applying this procedure $l$ times and taking into the account inequalities (7) and (8), we obtain that if $l$ is sufficiently large, and $\delta$ is sufficiently small, then

$$
\dot{V}(\mathbf{x}(t)) \leq-\frac{\tilde{a}}{2} \sum_{s=1}^{m}\left\|\mathbf{z}_{s}(t)\right\|^{\gamma_{s}+\mu_{s}-1} .
$$

Thus, the Lyapunov function (6) satisfies all the assumptions of Theorem 4.2 from [4]. Hence, the zero solution of (1) is asymptotically stable. This completes the proof.

\section{Example}

Let system (1) be of the form

$$
\dot{z}_{1}(t)=-z_{1}^{3}(t-\tau)+8 z_{2}^{\alpha}(t-\tau), \quad \dot{z}_{2}(t)=-z_{2}^{9}(t-\tau)+\beta z_{1}^{5}(t-\tau),
$$

where $z_{1}(t), z_{2}(t) \in \mathbb{R} ; \tau>0$ is a constant delay; $\alpha>1$ is a rational with the odd denominator, and $\beta>0$.

In this case, inequalities (4) can be written as follows

$$
\frac{3}{\alpha} \leq \frac{h_{2}}{h_{1}} \leq \frac{5}{9}
$$

Thus, the parameter $\alpha$ should satisfy the condition $\alpha \geq 27 / 5$.

Choose Lyapunov functions for the isolated delay free subsystems

$$
\dot{z}_{1}(t)=-z_{1}^{3}(t), \quad \dot{z}_{2}(t)=-z_{2}^{9}(t)
$$

in the form $V_{1}\left(z_{1}\right)=z_{1}^{8} / 8, V_{2}\left(z_{2}\right)=z_{2}^{10} / 10$. By the use of these functions, construct the corresponding system (5). We obtain

$$
-\eta_{1}^{3}+8 \eta_{2}^{\alpha}<0, \quad-\eta_{2}^{9}+\beta \eta_{1}^{5}<0
$$

Applying Theorem 1, it is easy to show that if one of the following conditions

(i) $\alpha>27 / 5$;

(ii) $\alpha=27 / 5$, and $\beta<1 / 32$

is fulfilled, then the zero solution of (9) is asymptotically stable for any positive value of delay. 


\section{Acknowledgement}

This work was supported in part by the Saint Petersburg State University, project no. 9.38.674.2013, and by the Russian Foundation of Basic Researches, grant nos. 13-0100347-a and 13-01-00376-a.

\section{References}

[1] A. Yu. Aleksandrov and A. V. Platonov, Aggregation and stability analysis of nonlinear complex systems, J. of Math. Analysis and Appl. Vol. 342, 2 (2008) 989-1002.

[2] A. Yu. AleKsANDrov and A. P. ZHABKo, On the stability of the solutions of a class of nonlinear delay systems, Automation and Remote Control. Vol. 67, 9 (2006) 1355-1365.

[3] K. Gu, V. L. Kharitonov and J. Chen, Stability of Time-Delay Systems, Birkhauser, Boston, MA, 2003.

[4] J. K. HAle and S. M. Verduyn Lunel, Introduction to Functional Differential Equations, Springer-Verlag, New York, 1993.

[5] A. A. Kosov, On the stability of complex systems by the nonlinear approximation, Differential Equations. Vol. 33, 10 (1997) 1440-1442.

[6] F. MAZENC and S.-I. NiCULESCU, Lyapunov stability analysis for nonlinear delay systems, Syst. \& Control Lett. Vol. 42 (2001) 245-251.

[7] S. Niculescu, Delay Effects on Stability: A Robust Control Approach. Lecture Notes in Control and Information Science, Springer, New York, Berlin, Heidelberg, 2001.

[8] L. Rosier, Homogeneous Lyapunov function for homogeneous continuous vector field, Syst. \& Control Lett. Vol. 19 (1992) 467-473.

[9] D. D. Siliak, Decentralized Control of Complex Systems, Academic Press, New York, 1991.

[10] V. I. ZuBov, Methods of A. M. Lyapunov and Their Applications, P. Noordhoff Ltd., Groningen, 1964. 